\title{
Inductive learning of normal clauses
}

\author{
Christel VRAIN ${ }^{1}$, Lionel MARTIN \\ L.I.F.O., Rue Léonard de Vinci \\ B.P. 6759,45067 Orléans Cedex 2 \\ France \\ email: cv@univ-orleans.fr martin@univ-orleans.fr
}

\begin{abstract}
In this paper, we are interested in the induction of normal clauses. We consider here the well-founded semantics, based on a three-valued logics. The classical constraint: the learned program must cover the positive examples and reject the negative ones, can be too strong and we have defined a weaker criterion: we require that a positive (resp. negative) example is not considered as False (resp. True) by the learned program. This study has been applied in a framework in many points similar to the system FOIL.
\end{abstract}

\section{Introduction}

In this paper we are interested in the induction of normal programs. To our knowledge, only the systems FOIL and FOCL $[4,3]$ can learn any normal programs but the method is purely syntactic and they do not really address the problem of the semantics of the learned program and of its correction relative to the initial specification.

We have chosen to study this problem in a framework in many points similar to the system FOIL. The domain theory is expressed by ground unit clauses, the predicate to learn is also defined extensionally. Its definition can be complete or partial; it enables to study two kinds of learning: reformulation and induction of knowledge.

We do not consider here the information-based heuristic that guides the search in FOIL. We are interested in the comparison between the intended interpretation of a program and the semantics of a program that extensionally covers $[7,5]$ the positive examples and rejects the negative ones.

The system FOIL uses an operational semantics of negation by failure that has few interesting theoretical properties. We have used the well-founded semantics [6] and therefore we have changed the way the training sets were built.

\section{The learning framework}

The aim is to learn an intensional definition of a predicate $Q$, from positive and negative examples of it. As inputs, we give a finite set $\mathcal{D}$ (the Herbrand

\footnotetext{
${ }^{1}$ corresponding member of the Inference and Learning group, L.R.I., university of Paris South
} 
universe) and some predicates $R_{i}, 1 \leq \mathrm{i} \leq \mathrm{j}$, defined extensionally on $\mathcal{D}$. The extension of a k-ary predicate $R_{i}$ is specified by two sets $E_{R_{i}}^{+}$and $E_{R_{i}}^{-}$of ground atoms $R_{i}\left(t_{1}, \ldots, t_{k}\right) ; E_{R_{i}}^{+} \cup \neg E_{R_{i}}^{-}$represents the intended interpretation of $R_{i}$. In this paper, the definition of $R_{i}$ is total $\left(E_{R_{i}}^{+} \cup E_{R_{i}}^{-}=\mathcal{D}^{k}\right)$.

We note $E^{+}=\left(\cup_{1}^{j} E_{R_{i}}^{+}\right) \cup E_{Q}^{+}$and $E^{-}=\left(\cup_{1}^{j} E_{R_{i}}^{-}\right) \cup E_{Q}^{-}$

We use the same basic algorithm as that of FOIL, but when a new literal is added, we compute differently the new sets of substitutions $T_{i}^{+}$and $T_{i}^{-}$that satisfy the body of a clause.

Let $T$ be a set of substitutions, $L$ a positive literal and $E$ a set of ground atoms. We note:

$\mathcal{T}(T, L, E)$ the set of substitutions $\sigma^{\prime} \mid$ there exists a substitution $\sigma \in T$,

- $\operatorname{support}\left(\sigma^{\prime}\right)=\operatorname{support}(\sigma) \cup$ variables $(L)$,

- $\forall X \in \operatorname{support}(\sigma), \sigma(X)=\sigma^{\prime}(X)$,

- L. $\sigma^{\prime} \in E$.

At the beginning of a new clause, let POS be the set of positive examples, not previously covered and $L_{0}=Q\left(X_{1}, \ldots, X_{n}\right)$ be the head of the clause.

$T_{0}^{+}=\mathcal{T}\left(\{\right.$ Id $\}, L_{0}$, POS $) ; T_{0}^{-}=\mathcal{T}\left(\{\right.$ Id $\left.\}, L_{0}, E_{Q}^{-}\right) ;$

1 - when adding a positive literal $L_{i}$ to the body of the clause,

$T_{i}^{+}=\mathcal{T}\left(T_{i-1}^{+}, L_{i}, E^{+}\right)$and $T_{i}^{-}=\mathcal{T}\left(T_{i-1}^{-}, L_{i}, \overline{E^{-}}\right)$

2- when adding a negative literal $L_{i}=\neg L_{i}^{\prime}$,

$T_{i}^{+}=\mathcal{T}\left(T_{i-1}^{+}, L_{i}^{\prime}, E^{-}\right) ; T_{i}^{-}=\mathcal{T}\left(T_{i-1}^{-}, L_{i}^{\prime}, \overline{E^{+}}\right)$

\section{Complete definition of $Q$ and $R_{i}$}

We have formalized in [7] the bias developed in FOIL [4, 1], based on an ordering of $D$. We say that the learned program $\mathcal{P}$ verifies the stratification bias if we can find an index $\mathrm{k}$ such that for each ground instance of a clause of $\mathcal{P}: Q\left(c_{1}\right.$, $\left.\ldots, c_{n}\right) \leftarrow L_{1}, \ldots, L_{m}$ satisfying:

- for each positive literal $L_{i}, L_{i} \in E^{+}$,

- for each negative literal $L_{i}=\neg L_{i}{ }^{\prime}, L_{i}{ }^{\prime} \in E^{-}$, then for each literal $L_{i}=Q\left(c_{1}{ }^{\prime}, \ldots, c_{n}^{\prime}\right)$ or $L_{i}=\neg Q\left(c_{1}{ }^{\prime}, \ldots, c_{n}{ }^{\prime}\right), c_{k}>c_{k}$ '.

When the definitions of the predicates $Q$ and $R_{i}$ are total, we have the two following results.

- if the learned program $\mathcal{P}$ is definite and satisfies the stratification bias, then the set $E^{+}$is the least Herbrand model of $\mathcal{P}$.

- if the learned program $\mathcal{P}$ is a normal program and satisfies the stratification bias, then the set $E^{+} \cup \neg E^{-}$is the well-founded model of $\mathcal{P}$. 


\section{Partial definition of $Q$}

We consider now the case the predicate $Q$ is partially defined $\left(\mathcal{D}^{n}-\left(E_{Q}^{+} \cup E_{Q}^{-}\right)\right.$ $\neq \emptyset$ ). In the 3 -valued well-founded semantics, the semantics of $\mathcal{P}$ is defined by the set $M_{\mathcal{P}}^{+}$of the ground atoms that are True for $\mathcal{P}$ and the set $M_{\mathcal{P}}^{-}$of the ground atoms that are False for $\mathcal{P}$. (We note $M_{\mathcal{P}}^{u}$ the set of undefined ground atoms.)

We can require that the semantics of the learned program verifies the following condition:

1: (strong acceptability criterion) $\quad E^{+} \subseteq M_{\mathcal{P}}^{+}$and $E^{-} \subseteq M_{\mathcal{P}}^{-}$

i.e. each positive (resp. negative) example is True (resp. False) for $\mathcal{P}$.

The system FOIL can learn programs that do not satisfy this constraint. (An example is given in [7]). We have modified the way the sets $T_{i}^{+}$and $T_{i}^{-}$ were computed and we have defined a weaker condition on the learned program:

2: (weak acceptability criterion) $E^{+} \subseteq \overline{M_{\mathcal{P}}^{-}}$and $E^{-} \subseteq \overline{M_{\mathcal{P}}^{+}}$

i.e. none of the positive (resp. negative) examples can be False (resp. True) for $\mathcal{P}$. This condition must be associated to a bias on the learning process that prevents the building of clauses like $Q\left(X_{1}, \ldots, X_{n}\right) \leftarrow \neg Q\left(X_{1}, \ldots, X_{n}\right)$ that would trivially satisfy this constraint.

To achieve the condition (2), we can weaken the stratification bias given in section $3[7]$ but as it depends on an ordering of $D$, we prefer using a new bias, which finds - when there exists one - a well-founded ordering of $E_{Q}^{+}$. To do this, we build the AND/OR graph, $\mathcal{G}^{+}(\mathcal{P})$, of the positive recursive calls, defined by:

- the elements of $E_{Q}^{+}$are nodes of the graph,

- for each ground instance of a clause of $\mathcal{P}: Q\left(c_{1}, \ldots, c_{n}\right) \leftarrow L_{1}, \ldots, L_{p}$ that covers extensionally an element of $E_{Q}^{+}$, we create a virtual node $\mathrm{N}, \mathrm{OR}$-son of the node $Q\left(c_{1}, \ldots, c_{n}\right)$ and having for AND-sons, the positive literals $L_{i}$ of predicate $Q$.

We say that a node is finitely dependent if there exists a finite path from it to a leaf and that $\mathcal{P}$ satisfies the bias of positive recursive calls when all the elements of $E_{Q}^{+}$are finitely dependent nodes of $\mathcal{G}^{+}(\mathcal{P})$.

Result: If the learned program $\mathcal{P}$ satisfies the bias of positive recursive calls, then it satisfies the weak acceptability criterion. (The proof uses a proof method adapted to the well-founded semantics [2].)

Example: Let $\mathcal{D}$ be the domain $\{1,2,3,4,5,6\}$. All the definitions are total and the sets $E^{+}$are defined by:

$$
\begin{aligned}
& E_{r_{1}}^{+}=\{(1,2),(3,2),(2,3),(1,4),(4,5),(5,1),(5,4),(5,6),(6,4)\} \\
& E_{r_{2}}^{+}=\{(6)\} \\
& E_{q}^{+}=\{(1),(4),(5),(6)\}
\end{aligned}
$$

We assume that the learned program is $q(\mathrm{X}) \leftarrow r_{2}(\mathrm{X})$.

$$
q(\mathrm{X}) \leftarrow r_{1}(\mathrm{X}, \mathrm{Y}), q(\mathrm{Y})
$$


The first bias presented in section 3 cannot be applied since for every ordering of $\mathcal{D}$, there exists no index of stratification. All the nodes of $E_{q}^{+}$are finitely dependent nodes and then $\mathcal{P}$ is accepted. Here, the extensional definitions are total for all the predicates and $\mathcal{P}$ has a total well founded model (in this case, $\mathcal{P}$ satisfies also the "strong" acceptability criterion).

\section{Conclusion}

We have studied the semantics of the learned program and defined biases so that the program satisfies at least the weak acceptability criterion.

The bias of positive recursive calls has been extended to a bias of recursive calls to get programs that satisfy the strong acceptability condition. Moreover, this work can be applied even if the relations $R_{i}$ are partially defined.

\section{References}

[1] Cameron-Jones R.M., Quinlan J.R., 1993. Avoiding Pitfalls When Learning Recursive Theories. Proceedings of the Thirteen International Joint Conference on Artificial Intelligence, Chambéry, France, August 28 - September 3, 1993, Vol. 2, pp. 1044-1049.

[2] Ferrand G., Deransart P., 1992. Proof method of partial correctness and weak completeness for normal logic programs. Joint International Conference and Symposium on Logic Programming, Washington, OctoberNovember 1992.

[3] Pazzani M., Kibler D., 1992. The Utility of Knowledge in Inductive Learning. Machine Learning, Vol. 9, $\mathrm{N}^{\circ}$. 1, June 1992, Kluwer Academic Publishers, pp. 56-94.

[4] Quinlan J.R., 1990. Learning Logical Definitions from Relations. Machine Learning Journal, Vol. 5, Kluwer Academic Publishers, pp. 239-266.

[5] de Raedt L., Lavrac N., Dzeroski S., 1993. Multiple Predicate Learning. Proceedings of the Thirteen International Joint Conference on Artificial Intelligence, Chambéry, France, August 28 - September 3, 1993, Vol. 2, pp. 1037-1043.

[6] Van Gelder A., Ross K.A., Schlipf J.S., 1991. The well-founded Semantics for General Logic Program. Journal of the ACM, Vol. 38, No. 3, July 1991, 620-650.

[7] Vrain C., Martin L., 1993. Induction de clauses normales: application au système FOIL. Rapport de recherche LIFO, 93-6, university of Orléans. 